\title{
Determining the need for palliative care in the emergency department: A feasibility study
}

\author{
Amanda L. Hill ${ }^{* 1}$, Tonja M. Hartjes ${ }^{1}$, Jonathan A. Massey ${ }^{2}$ \\ ${ }^{1}$ School of Nursing, University of Florida, Gainesville, United States \\ ${ }^{2}$ School of Medicine, American University of Integrative Sciences, St. Maarten, Netherlands Antilles
}

Received: April 21, 2016

DOI: $10.5430 / \mathrm{cns} . v 4 \mathrm{n} 3 \mathrm{p} 25$
Accepted: May 15, 2016

Online Published: May 24, 2016

\begin{abstract}
Background: According to the Centers for Disease Control and Prevention (CDC), there are over 136 million emergency department (ED) visits annually with increasing use and overcrowding of the ED. The ED's fast paced, often aggressive treatment nature can provide considerable obstacles to achieving desired benchmarks of quality patient and family centered care that shows improved outcomes along with high levels of satisfaction. Recognizing the need for palliative care (PC) in emergency medicine to facilitate better care, the Center to Advance Palliative Care (CAPC) developed the Improving Palliative Care in Emergency Medicine (IPAL-EM) Project.

Methods: A retrospective review of an integrated data repository (IDR) was completed to determine the feasibility, design, and implementation of a PC protocol in the ED of a large, academic Level I Trauma Center. The IPAL-EM Project's recommendations were explored as we targeted trauma-alert and traumatic intracerebral hemorrhage (ICH) patients.

Results: The protocol identified 719 patient encounters over an eight month time-frame that would have triggered an ED initiated PC consultation. Using Meier's average per-patient per-admission net cost savings related to PC of $\$ 2,659$ per encounter, there was a potential for over $\$ 1.9$ million in savings for the facility.

Conclusions: PC has demonstrated numerous benefits in various healthcare settings. Studies describing PC in the ED are limited. Furthermore, its culture was not viewed as conducive to PC incorporation. However, future studies investigating the impact of PC on length of stay (LOS), symptom management, patient/family satisfaction, and costs of care is critical.
\end{abstract}

Key Words: Emergency department, Feasibility study, Palliative, Emergency department initiated palliative care, Improving Palliative Care in Emergency Medicine Project

\section{INTRODUCTION}

The Centers for Disease Control and Prevention (CDC) reports over 136 million emergency department (ED) visits annually in the United States, resulting in over 16 million admissions to the hospital. ${ }^{[1]}$ The ED is increasingly utilized since the implementation of the Affordable Care Act - which has added a net total of 16.9 million newly insured individuals, with 12.6 million of these individuals newly enrolled in Medicaid. ${ }^{[2]}$ EDs are often faced with problems of overcrowding. This problem is expected to continue to grow with increasing numbers of emergency visits, coupled with an aging, chronically ill population, and a shortage of primary care providers in the outpatient setting. ${ }^{[3]}$ With limited health care resources hospitals and providers alike must focus on finding prudent ways to provide quality, patient and family centered, cost effective care that heeds patient and family

*Correspondence: Amanda L. Hill; Email: amandalhill@ufl.edu; Address: School of Nursing, University of Florida, Gainesville, United States. 
goals. Additionally, providers have an ethical duty to ensure quality care and prevent harm. ${ }^{[4]}$

\subsection{ED patients' characteristics}

The CDC's most recent data available for leading causes of death in the United States are for 2013, reporting unintentional injury as the fourth leading cause of death for all age groups, the leading cause of death for ages 1-44 years old, and the third leading cause of death in ages 45-64 years old. ${ }^{[5]}$ It is estimated annually that 32.4 to 41 million people are treated in EDs for trauma with 2.3 million people being admitted to hospitals related to injury. ${ }^{[1,6]}$ Additionally, every six minutes an individual is either killed or permanently disabled, costing more than $\$ 400$ billion each yearmaking trauma the most expensive health condition in the United States. ${ }^{[7,8]}$ The types of traumatic injuries that occur are wide ranging. One of these includes intracerebral hemorrhage $(\mathrm{ICH})$, which has considerable effects on patient outcomes and significant financial aftermath. In the United States, ICH affects an estimated $12-15$ per 100,000 individuals, and over 20,000 people die due to $\mathrm{ICH}^{[9]}$

\subsection{ED initiated PC: A possible solution}

Strategies to improve patient and family satisfaction, overall hospital operations, and improve outcomes such as reducing morbidity, mortality, length of stay (LOS), and readmissions rates need to be incorporated into the culture and environment of the ED. ${ }^{[10]}$ A possible solution is the use of PC within the $\mathrm{ED}$, as $\mathrm{PC}$ has been shown to offer a variety of benefits to address areas of need such as decreasing costs, decreasing LOS, and increasing patient satisfaction. ${ }^{[10-12]}$ As shown in Table 1, there are numerous research supported benefits of PC.

Table 1. Research supported benefits of PC

- Reduces inpatient medical costs

- Reduces post discharge costs

- Decreases number of intensive care unit (ICU) admissions upon subsequent hospitalizations

- Decreases ICU LOS and hospital stays

- Facilitates better clinical outcomes

- Facilitates treatment completion

- Helps address and relieve physical, emotional, and psychosocial suffering

- Facilitates communication among families, patients, and healthcare providers (HCPs)

- Facilitates fluid and safe care transitions

- Aides patients and families with difficult decision making

- Increases the number of completed Advance Directives upon discharge

- Facilitates care in line with patients' wishes and values

- Improves patient and family satisfaction

- Increases HCP satisfaction
The Center to Advance Palliative Care (CAPC) explains:

"PC is specialized medical care for people with serious illnesses. It focuses on providing relief from the symptoms, pain, and stress of a serious illness. The goal is to improve quality of life for both the patient and the family... It is appropriate at any age and at any stage in a serious illness, and can be provided along with curative treatment" (para. 1). ${ }^{[13]}$

\subsection{Significance of $P C$ in the ED}

While hospitals have increasingly continued to incorporate PC, the ED has not reflected a similar increase. Sixty-seven percent of United States hospitals with 50 beds or more had PC programs at the end of 2014, as compared to 53\% in a 2008 report; $90 \%$ of hospitals with 300 beds or more were found to have PC programs. ${ }^{[14]}$ Previously the ED has not been considered a conducive environment for the integration of PC services, however benefits have started to emerge as to the potential impact of starting PC in the ED. ${ }^{[12,15,16]}$

Additionally, the emergence and reinforcement of the concept that curative treatment and palliation can and should occur simultaneously has PC being viewed applicable to a wider scope of health care settings. ${ }^{[17,18]}$ The Improving Palliative Care in Emergency Medicine (IPAL-EM) Project by the CAPC was created in response to recognizing that the ED provided a unique opportunity to initiate PC. It provides a framework for developing and implementing a PC program in the ED. ${ }^{[19]}$

Current care must be patient individualized, incorporate the support of families and caregivers, ensure safety and quality, and facilitate interdisciplinary cooperation and communication with all parties. ${ }^{[17]}$ Research has revealed that PC can assist in all of these areas. Patients and families report increased satisfaction with care and providers, care is aligned with patients' wishes, and also helps decrease costs of care with the utilization of PC. ${ }^{[20]}$ Formal studies regarding the feasibility and impact of incorporating PC are limited, and more research is needed. ${ }^{[10,21]}$

\section{METHODS}

\subsection{Study design}

This study explored the feasibility of developing a PC protocol in an ED in a large, academic Level-I Trauma Center, and its impact on patient care described. Article recommendations coupled with the IPAL-EM Project Steps were utilized along with key stakeholder input to develop the protocol trigger. ${ }^{[22,23]}$ 


\subsection{Setting}

The ED is situated within an academic, non-profit, Level-

I Trauma Center with over 900 inpatient beds within the southeastern United States. ${ }^{[24]}$ The state-designated Level I Trauma Center annually services over 2,000 trauma patients. It is a 66-bed ED - including six trauma/resuscitation rooms. In the most recent reported statistics, its ED had over 75,000 visits with more than 41,000 admissions. ${ }^{[24]}$ The ED has the only burn center in the region, and has a helipad landing site able to accommodate medical air evacuation transports. Additionally, it is an accredited chest pain center as well as an accredited primary and comprehensive stroke center. ${ }^{[25,26]}$ The study unit serves the adult and geriatric populations for 14 counties and also sees patients referred from Georgia and Alabama. ${ }^{[25,27]}$ Pediatric patients are seen at a separate, designated pediatric ED run by the same facility.

\subsection{Selection of the study population}

A realistic, beneficial option for the ED was a protocol triggering consults for the adult trauma-alert and traumatic ICH patient populations. While all ICH cases are not due to penetrating or non-penetrating head trauma for the following statistic, ICH has a 30-day mortality rate of 44\%. ${ }^{[9]}$ Due to the substantial impact traumatic injuries and $\mathrm{ICH}$ have, coupled with key stakeholders expressing a need and interest to target these patient populations, trauma-alert and traumatic ICH were utilized as the populations for this feasibility study. The goal was to create an ED initiated PC consult protocol without significantly increasing nursing workload - particularly avoiding increasing documentation.

\subsection{Assessing the environment}

Initially, the facility's PC experts, including the PC medical director, advanced registered nurse practitioner (ARNP), and faculty expert were interviewed regarding an ED initiated PC protocol. A review of literature was completed, outlined, and discussed with relevant key stakeholders and champions. Building upon prior efforts in other EDs along with the IPALEM Project Steps, more applicable strategies were able to be gleaned to design the protocol.

Local PC and hospice resources were identified. The ED had access to an inpatient PC team, and the facility was working to establish an in-house PC unit. The ED and facility have a partner community hospice provider that provides home and residential services. There is around the clock chaplaincy support either in-house or on-call, but the ED does not have a full-time designated chaplain. Social service support was available, but the ED itself did not have a designated social service support team. Management and nursing voiced that a full-time social worker would greatly facilitate any PC protocol implementation and future changes. The ED does not treat pediatric patients, but the facility has a separate pediatric ED and its child life specialist resources can be called upon if warranted.

A needs assessment was completed utilizing a brief strengths, weaknesses, opportunities, threats (SWOT) analysis outlined in Table 2. ${ }^{[19,22,23]}$

Table 2. SWOT analysis

\footnotetext{
Strengths/Support

- Key stakeholders buy-in voiced.

- Champions identified and voiced their support of PC protocol in the ED.

- $\quad$ PC service looking for opportunities to expand.

Weaknesses/Barriers (ED and PC; Cultural and Environmental)

- $\quad$ Lack of full-time, ED specific social workers.

- High patient volume area with limited resources (money, time, personnel, etc.) where currency is speed.

- Environment with frequent distractions/interruptions not typically conducive to sensitive PC discussions with patients/families.

Opportunities/Expected Outcomes

- $\quad$ Collaborate with nursing educators in ED to strengthen PC bundles already in place.

- Collaborate with ED staff champions to tailor an ED PC protocol to specific needs.

- $\quad$ Communicate with ED the purpose of the PC protocol and potential ED specific benefits.

- Increase in percentage of ED patients/families reporting excellent pain/symptom management.

\section{Threats/Challenges}

- Without full-time social work or trained nursing support, ED has voiced that it will face various obstacles to implementing a protocol requiring additional charting (such as a function assessment score utilizing a Karnofsky Score).

- Insufficient funding for needed social worker and/or nurse navigator for ED PC protocol implementation process.

- Insufficient time/resources for adequate follow-up to measure outcomes for analysis of implementation success.
} 
During this time, financial and facility resource changes negatively impacted the support and implementation of a new protocol. Thus a feasibility study was conducted to determine the impact of an ED initiated PC consult protocol. A review of the electronic health record (EHR) and department database to determine the number of trauma-alert patients and traumatic ICH patients who were treated from $01 / 01 / 2014$ to $08 / 31 / 2014$ was completed to aid the PC team in projecting the estimated increased number of PC consults. A current review of the average number of ED initiated PC consults would have been beneficial in helping estimate the anticipated increase in PC consults and potentially help demonstrate the necessity of the project. Formal data of ED initiated PC consults were not able to be obtained. Informal interviews with nurses and the PC team revealed that orders placed for ED initiated PC consults were very rare.

At the time, the ED did not have any formal protocols or procedures related to initiating PC consults. Two designated $\mathrm{PC}$ resource binders - created by the ARNP on the PC team - are available for health care providers (HCPs) with contact information including but not limited to relevant support services, local hospice information, volunteer services, and the PC team contact information. Nursing voiced that these binders were helpful in many patient cases, but that typically they ended up calling the PC team's ARNP for assistance. As the in-patient PC team does not provide in-house 24 hours aday coverage, if these cases requiring PC's assistance present outside in-house coverage hours there can be delays in consultation.

\section{Anticipate potential obstacles}

Potential obstacles to the project's implementation were anticipated in the SWOT analysis. Identified areas included the following:

- Budgetary constraints related to:

Provider and nursing department education; Social worker or nurse navigator for pilot program implementation; EHR alterations made by information technology; Follow-up/tracking of customer service metrics and patient outcomes

- Potential push-back related to the project from department providers, nursing, surgeons, neuromedicine, neurosurgery, and/or Trauma Services Team

- Limited and/or skewed provider and/or nursing knowledge and understanding of PC and/or patient populations project is targeting

- Limited utilization of EHR PC trigger for ED initiated PC consults

\subsection{Developing a plan}

The SWOT analysis was continuously referenced while designing a plan. The integrated data repository (IDR) is run and maintained by the ED's affiliated university; the IDR houses de-identified patient information of the facility's patients' EHRs, which is able to be queried utilizing International Classification of Diseases, Ninth Revision, Clinical Modification (ICD-9-CM) codes. The IDR was queried to determine how many patients would qualify for PC consultation with the proposed protocol related to traumatic $\mathrm{ICH}$, but did not have a trauma-alert search term at the time of this study; the qualifying trauma-alert patient encounters over the same time period - $1 / 1 / 2014$ to $8 / 31 / 2014$ - were obtained from the facility's Trauma Services Manager. None of this data had any identifying patient information and no human subjects were part of this study, thus institutional review board approval was not required. ${ }^{[28]}$

\subsection{Trauma-alert patient encounters}

Patient encounters included the following:

- Designated trauma-alert patient presenting to the ED

- Patient cared for by Trauma Services Team

- Patient age 18 years or older

- Presentation to ED during time span of 01/01/2014 to $08 / 31 / 2014$

Five-hundred forty six patients were identified, which was $87.6 \%$ of the total (623 total) trauma-alert patients seen by the facility's ED in the designated time span.

\subsubsection{Traumatic ICH patient encounters}

Relevant traumatic ICH encounters using ICD-9-CM 853.0 (Other and unspecified intracranial hemorrhage following injury without mention of open intracranial wound) were queried and yielded 22 patients. To try and capture more traumatic ICH patient encounters, an additional query was conducted adding the ICD-9-CM 431 (ICH) to the search criteria. This query yielded 173 patients.

\subsubsection{All patient encounters for study population}

The combined total of patient encounters qualifying for the proposed PC protocol was 719 over an eight month period the 546 trauma-alert plus the 173 traumatic ICH.

\subsection{ED initiated PC consult protocol}

The aim was to create a quick, simple ED initiated PC consult protocol to provide a foundation for incorporating PC into the ED's culture. This initial trigger designed for the protocol focused on two specific patient populations - trauma-alert patients and traumatic ICH patients. Please see Figure 1 for a diagram of how patients would be screened as part of the admission process utilizing the EHR ED initiated PC consult 
protocol. If the treating provider was not the one who completed this screening tool and the automatic PC consultation is placed, a flagged alert will populate on the chart requiring the treating provider to acknowledge that this patient has met the screening criteria for $\mathrm{PC}$ consult. The treating provider will have the ability to cancel the automatic PC consultation through the EHR if the patient, or health care surrogate, refuses this service.

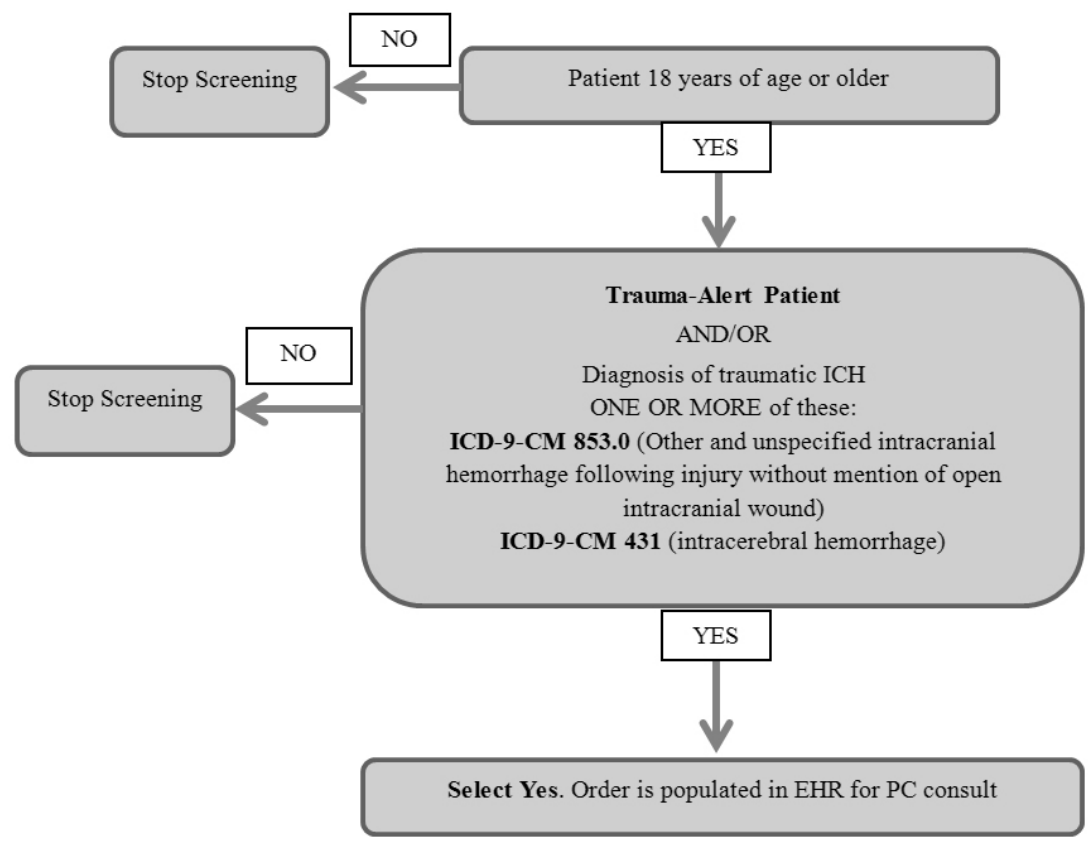

Figure 1. Screening utilizing the EHR ED Initiated PC consult protocol

The protocol for admitting documentation will have to be adjusted to incorporate the ED initiated PC consult protocol once the related EHR adjustments are completed. The modified protocol will incorporate the requirement that all EHR charts for patients 18 years and older have the trigger tool completed. The goal is to have this trigger tool completed in all EHR charts of patients 18 years and older within 30 minutes of being admitted to the ED. The protocol will state that nursing has the responsibility of completing this screening trigger tool in the protocol; any HCP, however, will have the charting capacity in the EHR to complete the screening trigger tool. At the time of this study, ICD-9-CM codes were being utilized, and would now have to be changed to the appropriate ICD-10-CM codes utilized by the EHR.

\section{Results}

\subsection{Outcome measures}

Upon completion of this feasibility study, it was noted that within an eight month timeframe 719 patients would have benefited from PC services in the ED. Examining relevant data - particularly comparing pre- and post-implementation outcomes - may help to guide further quality improvement projects at the facility and future research in the area of PC initiation and use in the ED.

\subsubsection{Study population characteristics}

The characteristics of the patients who end up meeting the ED initiated PC consult protocol criteria should be evaluated. Knowing the characteristics of this population may help reveal trends that could aid in results being able to be generalized to other patient populations.

\subsubsection{Pertinent data elements}

These data elements should be measured prior to implementation and at designated intervals after implementation:

- ED initiated PC consultation orders placed

- Mean ED initiated PC consults completed in ED

- Mean ED initiated PC consults completed outside of $\mathrm{ED}$

- Number of ED initiated PC consults cancelled due to patient/caregiver refusal

- Mean ED LOS in hours

- Mean LOS in days for admitted patients

- Patient mortality rates

- Hospital costs per patient

- ED discharge status (death in ED, admit-floor, admitintensive care unit [ICU], hospice referral-home or other site, long term care, home)

- Death within 72 hours of hospital admission (ICU vs. floor) 
- Completion rate of ED initiated PC consult trigger tool for ED patients:

Overall completion rate; Completion rate of goal time within 30 minutes of admission to ED

- Discharge disposition

Demonstrations of decreased LOS, improved costs, or improved patient mortality rates may provide a foundation for increased support of further study. Other areas to evaluate would include the impact of provided PC education, HCP and staff satisfaction, and patient and caregiver satisfaction pre- and post- implementation.

\section{Discussion}

From 01/01/2014 to 8/31/2014, 719 qualifying patient encounters were identified. Using Meier's PC average cost savings of $\$ 2,659$ per encounter for these 719 patients, over $\$ 1.9$ million may have been saved in an eight month period. ${ }^{[17]}$ Additionally, the facility's neurosurgical ICU began discussions of a PC protocol in its patient population after learning of this study. While the focus of this feasibility study was the implementation of a PC program in the ED, there has been considerable success for PC programs in other departments.

The limitations of this feasibility study are important to consider. As the IDR did not have a trauma-alert search term, the predicted number of qualifying patient encounters - and thus potential cost savings - is limited as some may be duplicate encounters. The largest limitation was the financial constraints at the facility hindering implementing a pilot program and funding necessary personnel. Additionally, the ED's educational budget was already being utilized for other programs.

\section{CONCLUSIONS AND RECOMMENDATIONS FOR FUTURE STUDY}

With the highest condition related expenditure for adults ages 18-64 being treatment of trauma-related disorders at $\$ 56.7$ billion, it is clear that these conditions and others that qualify for PC deserve considerable insight to help control costs. ${ }^{[29]}$ May et al. suggest that future studies of PC's economic impact "earlier in the care trajectory" are necessary, and research similar to this feasibility study provides an excellent opportunity for this (p. 1062). ${ }^{[1]}$ The limited, initial ED PC research has provided promising results. There are opportunities for EDs to partner with a local hospice for a Hospice Nurse Navigator to facilitate implementing a program. An ED initiated PC protocol has the potential to benefit not only patients and families but also health care facilities and HCPs while fostering delivery of quality care. ${ }^{[10,12]}$

\section{CONFLicts OF INTEREST Disclosure}

The authors declare they have no conflict of interest.

\section{REFERENCES}

[1] Centers for Disease Control and Prevention. Emergency Department Visits [Internet]. Atlanta: CDC/National Center for Health Statistics; 2014 Mar 29 [updated 2016 Feb 3; cited 2016 Apr 16]. Available from: http://www.cdc.gov/nchs/fastats/emergen cy-department.htm

[2] RAND Corporation. Health Coverage Grows Under Affordable Care Act [Internet]. Santa Monica (CA): RAND Corporation; 2015 May 6 [cited 2016 Apr 16]. Available from: http://www.rand.org/new s/press/2015/05/06.html

[3] American College of Emergency Physicians. ER Visits Up Since Implementation of Affordable Care Act [Internet]. 2014 May 21 [cited 2015 Mar 1]. Available from: http://newsroom. acep.org/2014-05-21-ER-Visits-Up-S ince-Implementation-of-Affordable-Care-Act

[4] Beauchamp T, Childress J. Principles of biomedical ethics. 7th ed. New York: Oxford University Press; 2013

[5] CDC/National Center for Health Statistics. Health, United States, 2014 with Special Features on Adults Aged 55-64 [Internet]. Hyattsville (MD): CDC/National Center for Health Statistics; 2015 May 1 [cited 2016 Apr 16]. Available from: http://www.cdc.gov/nc hs/data/hus/hus14.pdf

[6] National Trauma Institute. Trauma Statistics [Internet]. 2014 Feb 1 [cited 2016 Apr 16]. Available from: http://www.nationaltrau mainstitute.org/home/trauma_statistics.html

[7] Centers for Disease Control and Prevention. Key Injury and Violence Data [Internet]. Atlanta: Centers for Disease Control and Prevention; 2015 Sep 30 [updated 2015 Sep 30; cited 2016 Apr 16]. Available from: http://www.cdc.gov/injury/wisqars/overview/ key_data.html

[8] University of South Alabama Medical Center. USA Trauma Center [Internet]. USA Health System. c2016 [cited 2016 Apr 16] Available from: http://www. usahealthsystem.com/usa-tra uma-center

[9] Liebeskind D. Intracranial Hemorrhage [Internet]. Medscape. 2015 Oct 14 [cited 2016 Apr 16]. Available from: http://emedicine. medscape.com/article/1163977-overview\#a0101

[10] Quest T, Herr S, Lamba S, et al. Demonstrations of clinical initiatives to improve palliative care in the emergency department: a report from the IPAL-EM Initiative. Ann Emerg Med [Internet]. 2013; 61(6): 661-67. PMid: 23548402. http://dx.doi.org/10.1016/j.ann emergmed.2013.01.019

[11] May P, Normand C, Morrison R. Economic impact of hospital inpatient palliative care consultation: review of current evidence and directions for future research. J Palliat Med [Internet]. 2014;17(9): 1054-63. PMid: 24984168. http://dx.doi.org/10.1089/jpm .2013 .0594 
[12] Smith S, Brick A, O'Hara S, et al. Evidence on the cost and costeffectiveness of palliative care: a literature review. Palliat Med [Internet]. 2013; 28(2): 130-50. PMid: 23838378. http://dx.doi.org $/ 10.1177 / 0269216313493466$

[13] Center to Advance Palliative Care. What Is Palliative Care? [Internet]. Center to Advance Palliative Care; c2012 [cited 2016 Apr 16]. Available from: https://getpalliativecare.org/whatis/

[14] Dumanovsky T, Augustin R, Rogers M, et al. The growth of palliative care in U.S. hospitals: a status report. J Palliat Med [Internet]. 2015; 19(1): 8-15. PMid: 26417923. http://dx.doi.org/10.1089/j pm. 2015.0351

[15] Glajchen M, Lawson R, Homel P, et al. A rapid two-stage screening protocol for palliative care in the emergency department: a quality improvement initiative. J Pain and Symptom Manage [Internet]. 2011; 42(5): 657-62. PMid: 22045368. http://dx.doi.org/10. 1016/j.jpainsymman. 2011.06.011

[16] Grudzen C, Richardson L, Hopper S, et al. Does palliative care have a future in the emergency department? Discussions with attending emergency physicians. J Pain and Symptom Manage [Internet]. 2012; 43(1): 1-9. PMid: 21802899. http://dx.doi.org/10.1016/j·j painsymman.2011.03.022

[17] Meier D. Increased access to palliative care and hospice services: opportunities to improve value in health care. Milbank Q [Internet]. 2011; 89(3): 343-80. PMid: 21933272. http://dx.doi.org/10. 1111/j.1468-0009.2011.00632.x

[18] World Health Organization. WHO Definition of Palliative Care [Internet]. WHO. c2016 [cited 2016 Apr 16]. Available from: http: //www.who.int/cancer/palliative/definition/en/

[19] Center to Advance Palliative Care. Improving Palliative Care in Emergency Medicine: Improvement Tools [Internet]. Center to Advance Palliative Care. c2014 [cited 2015 Apr 1]. Available from: https://www.capc.org/ipal/ipal-emergency-medicine/

[20] Worldwide Palliative Care Alliance. Global Atlas of Palliative Care at the End of Life [Internet]. Worldwide Palliative Care Alliance; c2014 [cited 2016 Apr 16]. Available from: http: //www . who. in t/nmh/Global_Atlas_of_Palliative_Care.pdf

[21] Wu F, Newman J, Lasher A, et al. Effects of initiating palliative care consultation in the emergency department on inpatient length of stay.
J Palliat Med [Internet]. 2013; 16(11): 1362-67. PMid: 23971709. http://dx.doi.org/10.1089/jpm. 2012.0352

[22] Bryant E, Quest T, DeSandre P, et al. Getting Started: Organizing an ED Palliative Care Initiative: A Technical Assistance Monograph from the IPAL-EM Project [Internet]. Center to Advance Palliative Care. c2011 [cited 2015 Apr 1]. Available from: http://ipal.cap c.org/downloads/ipal-em-getting-started.pdf

[23] DeSandre P, Stone S, Quest T, et al. Four Things to do in a Week: Integrating Palliative Care Services Into the Emergency Department [Internet]. Center to Advance Palliative Care. c2011 [cited 2015 Apr 1]. Available from: http://ipal.capc.org/downloads/ipal -em-four-things-to-do-in-a-week.pdf

[24] U.S. News and World Report. UF Health Gainesville [Internet]. U.S News and World Report LP. c2016 [cited 2016 Apr 16]. Available from: http://health.usnews.com/best-hospitals/a $\mathrm{rea} / \mathrm{fl} / \mathrm{shands-at-the-university-of-florida-6390283}$

[25] UF Health. UF Department of Emergency Medicine: About Us [Internet]. Gainesville (FL): University of Florida Health; c2016 [cited 2016 Feb 1]. Available from: http://emergency.med.ufl.edu/ about-us/

[26] UF Health. UF Health Shands Comprehensive Stroke Center [Internet]. Gainesville (FL): University of Florida Health; c2016 [cited 2016 Feb 1]. Available from: http://stroke.ufhealth.org/ab out/

[27] UF Health. UF Health Shands Emergency Room / Trauma Center [Internet]. Gainesville (FL): University of Florida Health; c2016 [cited 2016 Feb 1]. Available from: https://ufhealth.org/uf-healt h-shands-trauma-center

[28] U.S. Food and Drug Administration. Regulatory Information [Internet]. Silver Spring (MD): U.S. Food and Drug Administration; c2016 [cited 2016 Jan 1]. Available from: http://www.fda.gov/Regu latoryInformation/Guidances/ucm126420.htm

[29] U.S. Department of Health and Human Services. Statistic Brief \#471: Top Five Most Costly Conditions among Adults Age 18 and Older, 2012: Estimates for the U.S. Civilian Noninstitutionalized Population [Internet]. Rockville (MD): Agency for Healthcare Research and Quality, c2015 [cited 2016 Feb 1]. Available from: http://meps.ahrq.gov/mepsweb/data_files/publi cations/st $471 /$ stat 471 .shtml 